\title{
Placental fusion in a dichorionic-diamniotic IVF twin pregnancy - case presentation
}

\author{
Andreea CALOMFIRESCU-AVRAMESCU ${ }^{1}$, Mihaela DEMETRIAN ${ }^{1}$, \\ Georgeta GRECU ${ }^{1}$, Vlad DIMA ${ }^{1}$, Ioan GHERGHINA ${ }^{2}$ \\ ${ }^{1}$ Filantropia Hospital, Bucharest, Romania \\ 2"Alessandrescu-Rusescu" National Institute for Mother and Child Health, \\ Bucharest, Romania
}

\begin{abstract}
Twin pregnancies have an increased incidence in recent years, especially due to the development of assisted human reproduction techniques. Although most of the in vitro fertilization pregnancies are dichorionic tasks, exceptions may also occur following the placental fusion process. Twin pregnancies in which the fusion process occurs associate the complications of monochorial pregnancies such as prematurity, growth discordance between the twins, twin-to-twin transfusion syndrome, twin reversed arterial perfusion, with higher perinatal mortality and morbidity. In recent years, most studies have focused on correlations between the macro and microscopic aspects of the placenta and the associated complications.
\end{abstract}

Keywords: placental fusion, dichorionic-diamniotic twin pregnancy, twin-to-twin transfusion syndrome

\section{INTRODUCTION}

The development of assisted human reproduction techniques has led to an increase in the incidence of twin pregnancies. Twin pregnancies can be monozygotic, derived from a single egg which can be monochorionic-monoamniotic or monochorionic-diamniotic, genetically identical and same-sex twins, or dizygotic, each embryo from another egg, dichorial-diamniotic, different twins. Monochorionic pregnancies are associated with more complications than dichorionic $(1,2)$.

Complications of twin pregnancies are either associated with the mother such as gestational diabetes, spontaneous abortion, pregnancy-induced hypertension and preeclampsia, or associated with the fetuses: prematurity (average gestational age is about 35-36 weeks for twins), intrauterine growth restriction on both embrions or only one of them, congenital malformations, chromosomal abnormalities, twin-to-twin transfusion syndrome and others (3).

The prematurity associated with twin pregnancies has been an intensely debated issue in the literature because it associates an increased rate of perinatal mortality and requires specialized therapt in neonatal intensive care unit. In recent years, the extension of pregnancy as close as possible to term is being attempted (4).

Twin-to-twin transfusion syndrome (TTTS) is a rare complication of monochorionic twin pregnancies (between 10 and 20\%), but with increased mortality and morbidity. The pathophysiological mechanism involves the development of a discrepancy in placental blood flow between the two fetuses, due to the presence of placental vascular anastomoses, one being the donor and the other the recipient. The number and type of anastomoses (arterial-to-arterial, venous-to-venous, or arterial-to-venous) determine the severity of the case $(5,6)$. 


\section{AIM}

Our aim is to present a case of a twin pregnancy, dichorionic-diamniotic, obtained by IVF, in which, after birth, the placenta had characteristics of a unique and fused mass.

\section{MATERIAL AND METHODS}

It is a case report in which the placental macroscopic changes of a dichorionic-diamniotic twin pregnancy are presented.

\section{CASE REPORT}

We present the case of a caucasian woman, with no previous births, 36-year-old without significant personal pathological history, normal BMI, non-smoking, higher studies, no exposure to toxic environment, normal blood pressure, with twin pregnancy obtained through in vitro fertilization. During pregnancy the patient received treatment with Clexane and folic acid. The double test showed no chromosomal abnormalities. Also TORCH test was negative, the usual blood test analyses within normal limits and the cultures of urine and cervix did not isolate pathogenic germs. The fetal morphology ultrasound from the first trimester was within normal limits for the gestational age with harmonic development of both fetuses.

The patient was monitored weekly with measurements of Doppler flow at the level of the umbilical artery and venous duct, also of the amount of amniotic fluid and the biometric parameters. The pregnancy was considered dichorionic-diamniotic without associating signs of twin-to-twin transfusion syndrome (no single placental aspect, same sex on both sides - in this case being boy and girl, without polyhydramnios in one twin or oligohydramnios in the other, without discrepancies between the appearance of the umbilical cords). At 24 weeks of gestation, complete corticosteroid therapy was administrated due to high-risk delivery that was expected of a premature birth.

She was hospitalized at 29 weeks in „Filantropia" Hospital for the spontaneous onset of labor with rare painful uterine contractions. The gynecological examination revealed a dilatation of about $5 \mathrm{~cm}$, intact membranes, without bleeding or leukorrhea. The cardiotocographic examination presented a non-stress test with normal variability and normal heartbeat on both fetuses (141 beats per minute, 156 beats per minute). The vascular resistances on the middle cerebral artery, the um- bilical artery and the venous duct were within normal limits only in one fetus, in the other the vascular resistances were increased, in the middle cerebral artery it was 0.70 , in the umbilical artery 0.71 and in the venous duct 0.84 . Doppler ultrasound performed before birth revealed a more hyperecogenic placenta than the other one, with a reduced vascular color signal.

After finishing the investigations, at 29 weekes and one day, it was decided to finish the pregnancy by cesarean section due to the fragility of the fetuses and low resources to face a labour and natural birth. Two living newborns were extracted, twin I was a girl of 1,200 grams ( $50 \%$ percentile) IA $6 / 7$, twin II was a boy of 1,100 grams ( $45 \%$ percentile) IA 5/6/7.

The macroscopic examination of the placenta showed a single mass with a monochorionic appearance, although they were dichorionic-diamniotic, a normal weight of about 490 grams, an umbilical cord of about $40 \mathrm{~cm}$ each with normal insertions. Both sides of the placenta (twin I and twin II) were very different on the maternal side. The paler part belongs to twin II while the darker and congested part to twin I. Placenta showed maternal vascular malperfusion causing decreased blood flow to twin II.

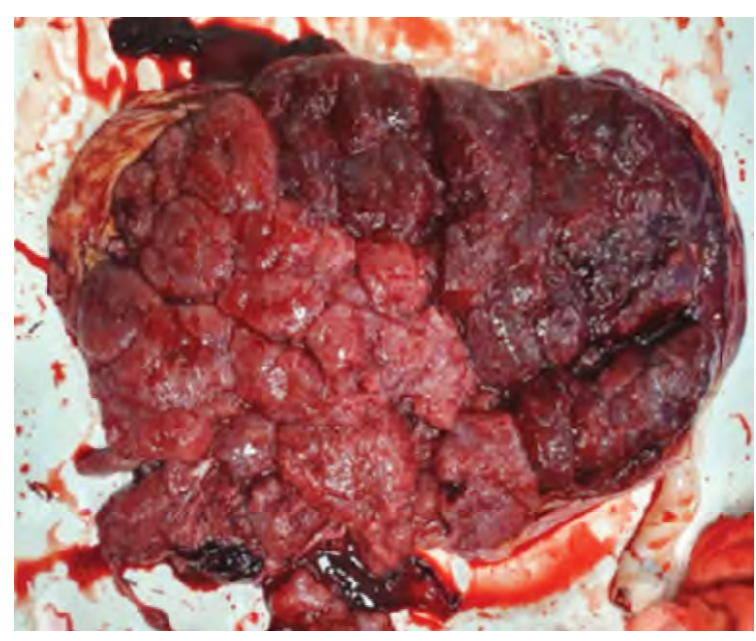

FIGURE 1. Macroscopic aspect of the placenta (the pale is from the twin II, the congested part is from the twin I)

First twin with the placenta who presented a normal macroscopic and microscopic appearance, had a favorable evolution with a moderate perinatal asphyxia, acrocyanosis, superficial respiration, hypotonia, absent archaic reflexes. In contrast, the second twin with the pale placenta presented severe perinatal asphyxia with generalized cyanosis, secondary apnea, hypotonia, absent archaic reflexes. 
Immediately after birth, both newborns were placed under radiant heat and mechanically ventilated with infant-T-piece resuscitator for alveolar recruitment. They were admitted to the neonatal intensive care unit where they were placed in closed incubators with thermoneutral point, and both mechanically ventilated (first twin in a non-invasive mode with nasal continous positive airway pressure for 24 hours, and the second twin in a invasive mode with synchronised intermittent mandatory ventilation and pressure support ventilation and volume targeted mode for 72 hours, then in the high flow nasal canula mode for 3 days). They received a single dose of surfactant (200 mg/kgc/dd) by the LISA method (twin I) and respectively, the classical method (twin II), parenteral partial nutrition on percutaneous central venous catheters and dopamine for hypotension.

The newborns were fed by gavage with mother milk, with good digestive tolerance and ascending weight curve. They needed phototherapy for jaundice, vitamin $D$ for osteopenia of prematurity and prophylactic therapy with iron for anemia of prematurity. They were transferred to the premature compartment at 21 days of life and released at 46 days with the weight of 2,540 grams twin I and 2,430 grams twin II.

Laboratory investigations initially showed leukocytosis with neutrophils, without anemia, without thrombocytopenia, positive inflammatory samples, then normalized. Hematocrit in twin I was $52.2 \%$ and hemoglobin $17.10 \mathrm{mg} / \mathrm{dl}$, and in twin II hematocrit $51.3 \%$ and hemoglobin 17.10 $\mathrm{mg} / \mathrm{dl}$. They did not require blood transfusions during hospitalization. They presented stage I retinopathy but without ophthalmological surgery. The transfontanelar ultrasound scans were adequate for the gestational age without significant changes in twin I, whereas in twin II the resistance index is slightly increased 0.72 .

\section{DISCUSSIONS}

Dichorionic twin pregnancies associate a lower rate of obstetric and neonatal complications compared with mono-chorionic ones. Dichorionic pregnancies in which placental fusion can occur may associate the complications of monochorionic pregnancies $(6,7)$. Although the pathophysiological mechanisms of the onset of complications differ, in both may occur: preterm birth, significant differences between birth weights, twin-to-twin transfusion syndrome or twin reversed arterial perfusion only due to placental anastomoses, genetic or malformative syndromes in both types of

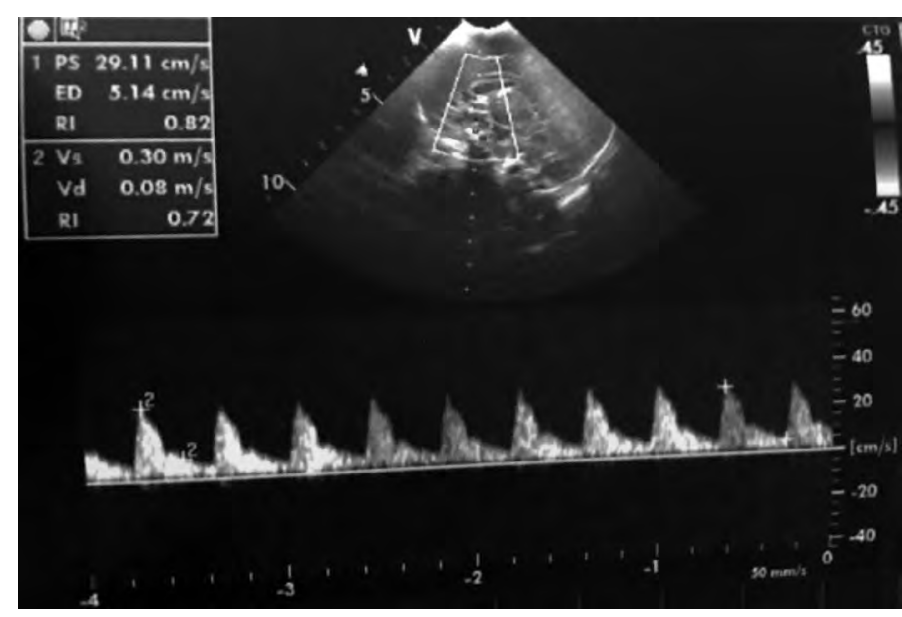

FIGURE 2. Transfontanela ultrasound: the resistance index in twin II

pregnancy. In our case, the pregnancy was dichorial-diamniotic but with placental fused with minimal vascular anastomoses. Even though twin pregnancies are associated with a higher rate of perinatal mortality, many studies have shown that monochorionic pregnancies have an even greater risk $(8,9)$.

Although the discordance of placental echogenicity associated with Doppler signal changes is an argument in the diagnosis of twin-to-twin transfusion syndrome, it can also occur in "stuck twin syndrome" (10). In the case presented the twin pregnancy was not complicated with any of the syndromes, thus suggesting the accomplishment of the placental fusion towards the end of the pregnancy (11).

In recent years, more and more researchers have shown close links between severe hypoxia at birth and placental changes (12). In this case, the caesarean section performed at the first signs of fetal suffering, the degree of hypoxia in both newborns was mild and respectively moderated without significant impact on their evolution. Many studies have been carried out to determine as accurately as possible the time of birth to prevent prematurity complications (13).

According to previous studies $(9,14)$, there may be greater differences between twin birth weights, mainly in the case of fused placentas, compared to those with separate placentas. In the presented case, the weights are relatively similar, falling between the 45th and 50th percentiles for gestational age. Also, the insertion of the umbilical cords was not velamentous. In a 2007 study by Suzuki S. an attempt was made to demonstrate the link between placental fusion and in vitro fertilization, but there was insufficient scientific evidence (7). 


\section{CONCLUSIONS}

Finally, following the clinical and paraclinical evidences of the presented case, we can say that the dichorionic-diamniotic twin pregnancy with macroscopic aspect of placental fusion and minimal placental anastomoses (which are specific to twin-to-twin transfused syndrome and twin reversed arterial perfusion) did not determine the occurrence of obstetric or neonatal complications except to a small extent. Newborns have only associated the complications of prematurity in mild forms.

Conflict of interest: none declared Financial support: none declared

\section{REFERENCES}

1. Luo L, Cai B, Jie HY, Gao Y, Chen M, Zhou $C Q$, Wang $Q$. Influence of spontaneous fetal reduction on dichorionic diamniotic twin pregnancy outcomes after in vitro fertilization: A large-sample retrospective study. J Matern Fetal Neonatal Med. 2019 Jun; 32(11):1826-1831.

2. Suzuki S, Murata T. The influence of assisted reproductive technology on growth discordance in dichorionic twin pregnancies. Fetal Diagn Ther 2007; 22:372-376.

3. Oloyede OA, Iketubosin F, Bamgbopa K et al. Spontaneous fetalreduction and early pregnancy complications in multiple pregnancies following in vitro fertilization. Int J Gynaecol Obstet. 2012;119(1):57-60.

4. Park YH, Kim YN, Im DH, Kim DH, Byun JM, Jeong DH, Lee KB, Sung MS. Neonatal outcomes between discordant monochorionic and dichorionic twins. J Matern Fetal Neonatal Med. 2019 Aug 28:1-9.

5. Peng R, Zhou Y, Xie H-N, Zheng J, Xie Y-J, Yang J-B. MCDA twins with discordant malformations: submicroscopic chromosomal anomalies detected by chromosomal microarray analysis and clinical outcomes. Prenat Diagn. 2016 Aug; 36(8):766-74.

6. Kusanovic JP, Romero R, Gotsch F, Mittal P, Erez O, Kim JC, Hassan SS, Espinoza J, Lami Yeo. Discordant placental echogenicity: A novel sign of impaired placental perfusion in twin-twin transfusion syndrome? The Journal of Maternal-Fetal and Neonatal Medicine, January 2010; 23(1):103-106.

7. Suzuki, S. Impact of Perinatal Outcome of the Presence of Fused or Separate Placentas in Dichorionic Twins. Fetal Diagnosis and Therapy, 2009; 26(3):127-130.

8. Coutinho Nunes F, Domingues AP, Vide Tavares $\mathrm{M}$ et al. Monochorionic versus dichorionic twins: Are obstetric outcomes always different? Journal of Obstetrics and Gynaecology. 2016;36(5):598-601.

9. Jahanfar S, Lim K. Adverse maternal outcomes and birth weight discordance in twin gestation: British Columbia, Canadian data. International Journal Of Women's Health. 2017;9:871.

10. Foschini MP1, Gabrielli L et. al Vascular anastomoses in dichorionic diamnioticfused placentas. Int J Gynecol Pathol. 2003 Oct; 22(4):359-61.
11. Brendan Fitzgerald. Histopathological examination of the placenta in twin pregnancies. APMIS Journal Of Pathology, Microbiology And Immunology, July 2018, Volume 126, 626-637.

12. Nasiell J, Papadogiannakis N, Löf $E$, Elofsson F, Hallberg B. Hypoxic ischemic encephalopathy in newborns linked to placental and umbilical cord abnormalities. The Journal of Maternal-Fetal \& Neonatal Medicine. 2015; 29(5):721-726.

13. Nesa Asnafi, Zahra Basirat, Karimollah Hajian-Tilaki, Sarah Dadvar. Assessment of cervical length by transvaginal ultrasonography to predict preterm delivery in twin pregnancy. J Matern Fetal Neonatal Med, 2013; 26(14):1435-1438.

14. Céline Homatter, Pierre-Yves Robillard, Asma Omarjee, Chloé Schweizer, Malik Boukerrou, Fabrice Cuillier, Bérénice Doray, Hanitra Randrianaivo, Bénédicte BertautNativel \& Coralie Dumont. Discordant malformations in monochorionic twins: A retrospective cohort study in $\mathrm{La}$ Reunion Island. The Journal of MaternalFetal \& Neonatal Medicine. 2019;14767058. 